\title{
ON-THE-FLY AMBIGUITY RESOLUTION USING AN ESTIMATOR OF THE MODIFIED AMBIGUITY COVARIANCE MATRIX FOR THE GNSS POSITIONING MODEL BASED ON PHASE DATA
}

\author{
S. Cellmer \\ Institute of Geodesy \\ University of Warmia and Mazury in Olsztyn \\ Poland \\ e-mail: slawomir.cellmer@gmail.com
}

\begin{abstract}
On-the-fly ambiguity resolution (OTF AR) is based on a small data set, obtained from a very short observation session or even from a single epoch observation. In these cases, a classical approach to ambiguity resolution (e.g. the Lambda method) can meet some numerical problems. The basis of the Lambda method is an integer decorrelation of the positive definite ambiguity covariance matrix (ACM). The necessary condition for the proper performing of this procedure is a positive definiteness of ACM. However, this condition is not satisfied in cases of very short observation sessions or single epoch positioning if phase only observations are used. The subject of this contribution is such a case where phase-only observations are used in the final part of the computational process. The modification of ACM is proposed in order to ensure its positive definiteness. An estimator of modified ACM is a good ACM approximation for the purpose of performing the LAMBDA method. Another problem of short sessions (or a single epoch) positioning is the poor quality of the float solution. In this paper, a cascade adjustment with wide-lane combinations of signals L1 and $\mathrm{L} 2$ as a method of solving this problem is presented.
\end{abstract}

Keywords: GNSS data processing, ambiguity resolution, Lambda method

\section{INTRODUCTION}

Integer Least Squares Adjustment (ILSA) is the key to high-precision Global Navigation Satellite System (GNSS) positioning. The well-known classical approach to ILSA consists of three stages (Teunissen, 1995):

- Float solution -least squares (LS)adjustment without constraints- all parameters (also ambiguities) are real valued

- Ambiguity Resolution (AR) - search for the best candidates of the integer valued ambiguities

- Fixed solution - LS solution with known constant integer valued ambiguities obtained in the second stage.

Currently, one of the most applied methods of ILSA, is the Least Squares Ambiguity Decorrelation Adjustment (LAMBDA) method developed by Teunissen (Joosten, 2001; 
Joosten and Tiberius, 2002; Teunisssen, 1995). Teunisssen (1999) proved that LAMBDA method is optimal in the sense of maximizing the probability of correct integer estimation. In this method, an integer decorrelation transformation is applied before the search for integer ambiguities. Although the decorrelation in LAMBDA does not enhance the success rate of ILSA it is needed to improve the efficiency of the search process. A decorrelation procedure is carried out on the basis of an ambiguity covariance matrix (ACM) obtained from a float solution. As expressed in the title, the topic of this paper is OTF AR. It means that ACM for the integer decorrelation procedure must be obtained from a float solution of a very short session or even from a single epoch observation (Kashani et al., 2005). In this case, ACM is not positive definite, if phase observations only are used. This fact causes serious difficulties associated with the integer decorrelation procedure. One solution to this problem may be addition of code observations, e.g., as in the generalized least squares model proposed by Wielgosz (2011). However, that standard approach has some weaknesses. The code observations can be severely biased, e.g. due to multipath. Then the phase-only approach provides a more robust method. This contribution proposes to use an estimator of modified $\mathrm{ACM}$ to decorrelation procedure in a case of single epoch positioning. This estimator is in the form of a positive definite matrix, characterized by the desirable properties. The single epoch solution has the following advantages:

- No need of solving "cycle slip" problem

- Independence such a solution from previous epochs solutions

- An immediate solution in RTK mode- there is no initialization procedure

- Identical computational process in RTK mode and in static session.

The proposed approach is one of the methods of ill-posed problem resolution. In the past few years the AR, as an ill-posed problem, was presented in a number of papers (Ou and Wang, 2004; Shen and Li, 2007; Gui and Han, 2007; Li et al., 2010). Most researchers have attempted to solve this problem using regularization technique. Ou and Wang (2004) regularized the baseline part of the normal equations. Shen and Li (2007) proposed to regularize the ambiguity parameters. Gui and Han (2007) regularized baseline and ambiguities simultaneously. An interesting method of regularization parameter estimation was proposed by Li et al. (2010). In this contribution another method of solving ill-posed problem is proposed. An estimator of modified ACM is derived simulating the presence of an additional group of observations. Then this matrix is used as the base for LAMBDA method. The proposed in this contribution the modified ACM has better properties in comparison to the ACM obtained from standard approach. These better properties in the sense of decorrelation procedure efficiency imply from special way of the simulated observations weighting scheme.

The next section of the paper contains a description of forming an estimator of modified ACM that is positive definite. In Section 3, a simple method of OTF AR is presented. In this method, different linear combinations (LC) of L1 and L2 signals are applied. The process consists of consecutive ARs with the Lambda method for LCs with gradually reduced wavelengths. A numerical example is given in Section 4. Section 5 presents some results of the tests carried out on the basis of real data. In final part of the paper some conclusions are formulated. 


\section{AN ESTIMATOR OF THE MODIFIED AMBIGUITY VARIANCE-COVARIANCE} MATRIX

The observation equation of DD carrier phase reads:

$$
\mathrm{L}+\mathrm{V}=\mathrm{AX}+\mathrm{BN} \text {, }
$$

where:

$\mathrm{L}$ - vector of DD carrier phase observations in cycles

$\mathrm{V}$ - residuals vector in cycles

A - design matrix for real valued parameters (baseline components)

$\mathrm{X}$ - vector of real valued parameters $[\mathrm{m}]$

$\mathrm{B}$ - design matrix for ambiguities

$\mathrm{N}$ - ambiguity vector in cycles

It is assumed in this contribution that dimension of $A$ matrix is $n \times 3$, where $n$ is a number of observations. It means that there are only three real-valued parameters (coordinates). The atmosphere parameters are ignored or modelled and embodied in L vector. The LS solution of the equations (1) is obtained from the following normal equations:

$$
\left[\begin{array}{ll}
A^{T} P A & A^{T} P B \\
B^{T} P A & B^{T} P B
\end{array}\right]\left[\begin{array}{l}
X \\
N
\end{array}\right]=\left[\begin{array}{l}
A^{T} P L \\
B^{T} P L
\end{array}\right],
$$

where $\mathrm{P}$ is an observation weight matrix.

The solution of the equation (2) is:

$$
\left[\begin{array}{l}
X \\
N
\end{array}\right]=\left[\begin{array}{ll}
A^{\mathrm{T}} P A & A^{\mathrm{T}} P B \\
\mathrm{~B}^{\mathrm{T}} \mathrm{PA} & \mathrm{B}^{\mathrm{T}} \mathrm{PB}
\end{array}\right]^{-1}\left[\begin{array}{l}
\mathrm{A}^{\mathrm{T}} \mathrm{PL} \\
\mathrm{B}^{\mathrm{T}} \mathrm{PL}
\end{array}\right],
$$

Let's denote:

$$
\left[\begin{array}{ll}
A^{T} P A & A^{T} P B \\
B^{T} P A & B^{T} P B
\end{array}\right]^{-1}=\left[\begin{array}{ll}
P_{X X} & P_{X N} \\
P_{N X} & P_{N N}
\end{array}\right]^{-1}
$$

It can be easily verified that:

$$
\left[\begin{array}{ll}
\mathrm{P}_{\mathrm{XX}} & \mathrm{P}_{\mathrm{XN}} \\
\mathrm{P}_{\mathrm{NX}} & \mathrm{P}_{\mathrm{NN}}
\end{array}\right]^{-1}=\left[\begin{array}{ll}
\mathrm{Q}_{\mathrm{XX}} & \mathrm{Q}_{\mathrm{XN}} \\
\mathrm{Q}_{\mathrm{NX}} & \mathrm{Q}_{\mathrm{NN}}
\end{array}\right]
$$

where:

$$
\begin{aligned}
& \mathrm{Q}_{\mathrm{NN}}=\left[\mathrm{P}_{\mathrm{NN}}-\mathrm{P}_{\mathrm{NX}} \mathrm{P}_{\mathrm{XX}}^{-1} \mathrm{P}_{\mathrm{XN}}\right]^{-1} \\
& \mathrm{Q}_{\mathrm{XX}}=\mathrm{P}_{\mathrm{XX}}^{-1}+\mathrm{P}_{\mathrm{XX}}^{-1} \mathrm{P}_{\mathrm{XN}} \mathrm{Q}_{\mathrm{NN}} \mathrm{P}_{\mathrm{NX}} \mathrm{P}_{\mathrm{XX}}^{-1} \\
& \mathrm{Q}_{\mathrm{XN}}=-\mathrm{P}_{\mathrm{XX}}^{-1} \mathrm{P}_{\mathrm{XN}} \mathrm{Q}_{\mathrm{NN}} \\
& \mathrm{Q}_{\mathrm{NX}}=\mathrm{Q}_{\mathrm{XN}}^{\mathrm{T}}
\end{aligned}
$$

Matrix $\mathrm{Q}_{\mathrm{NN}}$ is an ambiguity covariance matrix that on the basis of (4) and (6) can be expressed in the following form:

$$
\mathrm{Q}_{\mathrm{NN}}=\left[\mathrm{B}^{\mathrm{T}} \mathrm{PB}-\mathrm{B}^{\mathrm{T}} \mathrm{PA} \quad \mathrm{A}^{\mathrm{T}} \mathrm{PA}{ }^{-1} \mathrm{~A}^{\mathrm{T}} \mathrm{PB}\right]^{-1}
$$

This matrix is the basis of the integer decorrelation procedure in the Lambda method. In the case of single epoch positioning, $\mathrm{B}$ is an identity matrix $(\mathrm{B}=\mathrm{I})$ and the ambiguity covariance matrix (10) is obtained as an inverse of the following matrix: 


$$
\mathrm{P}_{\mathrm{NNa}}=\mathrm{P}-\mathrm{PA} \mathrm{A}^{\mathrm{T}} \mathrm{PA}{ }^{-1} \mathrm{~A}^{\mathrm{T}} \mathrm{P}
$$

However, this matrix is not positive definite and therefore does not satisfy the condition required for the LAMBDA method. Nevertheless, matrix (11) can be replaced by its another estimator, which is positive definite. It is proposed here to estimate this matrix using simulated, additional observations. In this case, the matrix (10) can be expressed in the following form:

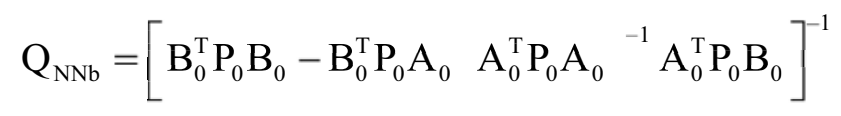

where $\mathrm{B}_{0}, \mathrm{~A}_{0}$ and $\mathrm{P}_{0}$ are the following block matrices:

$$
\left.\underset{2 \mathrm{n} \times \mathrm{n}}{\mathrm{B}_{0}}=\left[\begin{array}{c}
0 \\
\mathrm{n} \times \mathrm{n} \\
\underset{\mathrm{n} \times \mathrm{n}}{I}
\end{array}\right], \underset{2 \mathrm{n} \times 3}{\mathrm{~A}_{0}}=\left[\begin{array}{c}
\mathrm{A} \times 3 \\
\underset{\mathrm{n} \times 3}{\mathrm{~A}}
\end{array}\right], \mathrm{P}_{0}=\left[\begin{array}{cc}
\mathrm{P}_{\mathrm{a}} & \\
\mathrm{n} \times \mathrm{n} & \underset{\mathrm{n} \times \mathrm{n}}{\mathrm{P}}
\end{array}\right], \underset{\mathrm{n} \times \mathrm{n}}{(\mathrm{I}} \text { is } \mathrm{n} \times \mathrm{n} \text { identity matrix }\right)
$$

$\mathrm{P}_{\mathrm{a}}$ is a weight matrix of the simulated, additional observations. It is assumed here that design matrix A for simulated observations is the same as for real observations, and B is a null matrix for these observations. The weights of simulated observations must be small enough so that their impact on the results is negligible. On the other hand, they must assure the positive definiteness of the ambiguity covariance matrix. If we assume that the structures of the matrices $\mathrm{P}_{\mathrm{a}}$ and $\mathrm{P}$ are the same, then:

$$
\mathrm{P}_{\mathrm{a}}=\mathrm{cP},
$$

where $\mathrm{c}$ is a positive coefficient less than one $(0<\mathrm{c}<1)$.

Taking into account (13) and (14) the formula (12) can be rewritten as:

$$
\mathrm{Q}_{\mathrm{NNb}}=\left[\mathrm{P}-\mathrm{kPA} \quad \mathrm{A}^{\mathrm{T}} \mathrm{PA}{ }^{-1} \mathrm{~A}^{\mathrm{T}} \mathrm{P}\right]^{-1} \text {, where : } \mathrm{k}=\frac{1}{1+\mathrm{c}}
$$

It is proposed here to fix the coefficient $\mathrm{c}=0.01$ (the weights for simulated observations are 100 times smaller than weights for real data). Hence, the coefficient $\mathrm{k}$ equals 0.99 . No additional, theoretical studies were conducted to prove that this value of $\mathrm{c}$ is optimal, but it was tested that such value does not significantly affect the results and simultaneously it ensures the positive definiteness of the ACM (Cellmer, 2011a,b). It should be noticed, that the model (13) is in fact the same as if code observations were used along with the phase observations. The one significant difference is a way of the weighting of the simulated observations. The nominal standard deviation of code observations is about one hundred times lower than the nominal standard deviation of phase observations. Thus, in contrast to the model proposed in this contribution, in the standard model, the weights for code observations are 10000 times smaller than the weights of the phase observations. The $\mathrm{k}$ coefficient corresponding to such (standard) solution would be equal to 0.9999 . An additional advantage of the proposed model is that the code biases will not have an impact on the results.

In summary, the simulation of additional observations is equivalent to the use of the coefficient $\mathrm{k}$ as in (15). The formula (15) presents the estimator of modified ACM in the case of single epoch positioning. This matrix can be applied to the decorrelation procedure in the Lambda method.

\section{OTF AR USING LAMBDA METHOD IN CASCADE ADJUSTMENT}

In (Cellmer, 2010) it was shown that the graphical plot of the carrier phase LS Objective Function is an irregular surface. This is the case especially when the positioning is based on 
short sessions or on only a single epoch. Also extreme ionospheric conditions have adverse influence on the shape of LS Objective Function (Shagimuratov et al., 2002). Therefore, if float solution is not sufficiently close to the true solution, there is high risk of incorrect ambiguity fixing. The solution can be found in one of the local minima instead of in the global one, where the correct solution is. Therefore the approximate position in carrier phase process should be as good as possible. Reliable, approximate position can be obtained using Network Code DGPS Positioning (Bakula, 2010). However the accuracy can be still insufficient for classic carrier phase processing. Cellmer et al. (2010) proposed to solve this problem in MAFA method using cascade adjustment (CA). Cascade adjustment can be used as well in the classic approach with the LAMBDA method for AR (Henkel and Gunther, 2007). Instead of carrier phase data of only the L1 signal, the Linear Combinations (LC) with an integer coefficient and long wavelength can be applied. In this approach, the integer ambiguities of wide-lane combinations with successively reduced wavelengths are estimated. In this contribution, the three LC listed in Table 1 were chosen for the tests (Cellmer, 2009; Cellmer et al., 2010).

Table 1 Linear combinations of L1 and L2 signals

\begin{tabular}{|c|c|c|}
\hline $\mathbf{L C}_{\mathbf{i}, \mathbf{j}}$ & $\mathbf{i L 1}+\mathbf{j L 2}$ & $\boldsymbol{\lambda}[\mathbf{m}]$ \\
\hline $\mathrm{LC}_{-3,4}$ & $-3 \mathrm{~L} 1+4 \mathrm{~L} 2$ & 1.6281 \\
\hline $\mathrm{LC}_{1,-1}$ & $\mathrm{~L} 1-\mathrm{L} 2$ & 0.8619 \\
\hline $\mathrm{LC}_{10}=\mathrm{L}_{1}$ & $\mathrm{~L} 1$ & 0.1903 \\
\hline
\end{tabular}

Analyses of the theoretical properties of these combinations were carried out by Han and Rizos (1996), Urquhart (2009), Cocard and Geiger (1992). The computations were performed, starting from the LC with the longest wavelength and finishing with the L1 signal only. The position from the fixed solution at each stage of CA formed the basis for obtaining the float solution in the next stage. The large wavelength of LC simplifies integer ambiguity resolution in the first step of cascade adjustment. The a priori knowledge of ambiguities (with larger wavelength) from the previous step reduces the probability of wrong ambiguity fixing in the subsequent algorithm step.

\section{EXAMPLE BASED ON A SINGLE EPOCH}

The test of the presented algorithm was performed based on real data. The input data are listed in Tables 2 and 3. In the first row of Table 1, the coordinates of an apriori position are placed. This position was obtained using DGPS technique on the basis of code observations and one fixed reference station. In the second row, there are coordinates obtained from an 8 hour session processing using Bernese software (Dach et. al., 2007). These values are presented for the purposes of comparison with the single epoch processing results. In the first column of the Table 3 there are double-differenced geometric distances computed from apriori position coordinates. The second and third columns contain double differenced carrier phase observations of the signals L1 and L2. The fourth column contains the design matrix.

Table 2. A priori and 'true' coordinates

\begin{tabular}{|l|c|c|c|}
\hline & $\mathrm{X}[\mathrm{m}]$ & $\mathrm{Y}[\mathrm{m}]$ & $\mathrm{Z}[\mathrm{m}]$ \\
\hline a priori (DGPS) & 3717387.417 & 1256680.722 & 5011465.523 \\
\hline true & 3717386.066 & 1256680.646 & 5011465.539 \\
\hline
\end{tabular}


Table 3 Input data

\begin{tabular}{|r|r|r|rrr|}
\hline \multicolumn{1}{|c|}{ DD_dist [m] } & \multicolumn{1}{|c|}{ DD_L1[cycles] } & \multicolumn{1}{|c|}{ DD_L2[cycles] } & \multicolumn{3}{|c|}{ A } \\
\hline 7110.220 & 1306649.802 & 697089.627 & 0.0688 & 0.2350 & -0.0014 \\
-21107.951 & 770981.863 & 600050.122 & 1.1742 & -0.6315 & 0.1701 \\
-7214.561 & 5449276.945 & 4242415.741 & -0.3696 & -0.0758 & 0.7292 \\
-24238.136 & 2090537.500 & 411839.892 & 0.2210 & -0.7500 & 0.1875 \\
19892.307 & -2074454.151 & -1589927.638 & 0.3674 & 0.7018 & 0.1726 \\
-24521.234 & 4636828.079 & 2747848.515 & 0.6029 & -0.7752 & 0.0857 \\
\hline
\end{tabular}

The weight matrix was obtained as an inverse of the LC DD carried phase covariance matrix:

$$
\mathrm{P}=\mathrm{C}^{-1}
$$

with the following structure of matrix $\mathrm{C}$ :

$$
\mathrm{C}=\mathrm{m}^{2} \sigma^{2}\left[\begin{array}{cccccc}
4 & 2 & 2 & 2 & 2 & 2 \\
2 & 4 & 2 & 2 & 2 & 2 \\
2 & 2 & 4 & 2 & 2 & 2 \\
2 & 2 & 2 & 4 & 2 & 2 \\
2 & 2 & 2 & 2 & 4 & 2 \\
2 & 2 & 2 & 2 & 2 & 4
\end{array}\right]
$$

where:

$$
\begin{aligned}
& \sigma-\text { nominal accuracy of the carrier phase observation (one percent of the cycle, } \\
& \sigma=0.01 \text { ) } \\
& m-\text { LC noise, dependent on LC coefficients }\left(m=\sqrt{i^{2}+j^{2}} \text {, for } i, j \text { listed in Table } 1\right)
\end{aligned}
$$

The integer ambiguity resolution was carried out with LAMBDA method using the Peter Joosten Matlab function (Joosten, 2001). In Table 4, the results of the elaboration for two values of coefficient $\mathrm{k}$ are summarized. The value of $\mathrm{k}=0.99$ corresponds to a model proposed in this contribution while the value of $\mathrm{k}=0.9999$ corresponds to a standard model. The values $\Delta \mathrm{X}, \Delta \mathrm{Y}$ and $\Delta \mathrm{Z}$ are the residuals with respect to the 'true' coordinates at each stage of the cascade processing.

Table 4 The results of elaboration

\begin{tabular}{|l|rrr|rrr|}
\hline \multirow{2}{*}{$\mathrm{LC} \#$} & \multicolumn{3}{|c|}{$\mathrm{k}=0.99$} & \multicolumn{3}{c|}{$\mathrm{k}=0.9999$} \\
\cline { 2 - 7 } & \multicolumn{1}{|c|}{$\Delta \mathrm{X}$} & $\Delta \mathrm{Y}$ & $\Delta \mathrm{Z}$ & $\Delta \mathrm{X}$ & $\Delta \mathrm{Y}$ & $\Delta \mathrm{Z}$ \\
\hline DGPS & 1.352 & 0.076 & -0.016 & 1.352 & 0.076 & -0.016 \\
\hline $\mathrm{LC}_{-3.4}$ & -0.183 & 0.012 & 0.444 & 3.545 & -1.483 & -2.369 \\
\hline $\mathrm{LC}_{1,-1}$ & 0.016 & -0.013 & -0.055 & 2.856 & -0.868 & 2.475 \\
\hline $\mathrm{LC}_{1}$ & -0.007 & -0.010 & 0.003 & 1.585 & -1.016 & 1.925 \\
\hline
\end{tabular}

The graphical representations of these values are depicted in Fig 1. The horizontal axis on the level zero depicts the 'true' value of the coordinates. The blue, red and green lines show the residuals of the XYZ coordinates referenced to their 'true' values. The plot shows that in the case of $\mathrm{k}=0.99$ the solution of the cascade processing finally converges to the correct value even though the apriori position is almost $1.5 \mathrm{~m}$ away from true position. On the other hand the case of $k=0.9999$ did not give correct solution. .

$\mathrm{k}=0.99 \quad \mathrm{k}=0.9999$




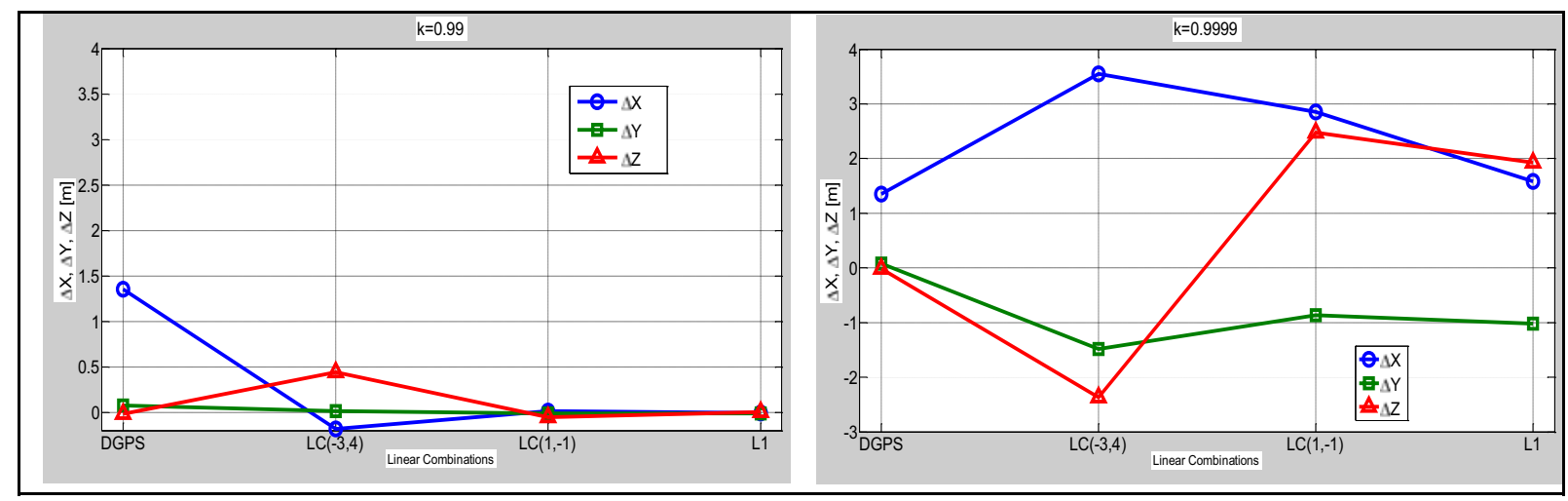

Fig. 1 The residuals referenced to the 'true' coordinates

\section{RESULTS FOR 120 INDEPENDENT EPOCHS}

In order to test the efficiency of the proposed algorithm, the real GPS data of two baselines was collected. Test surveys were performed on December 9th, 2008, on $30 \mathrm{~km}$ and $10 \mathrm{~km}$ baselines, with a 30-second sampling rate. Data sets of each baseline consisted of 120 epochs. The data was processed according to the proposed approach independently for each epoch. The ambiguity covariance matrix was formed according to formula (15), as a basis for the decorrelation procedure. Two various values of the $\mathrm{k}$ coefficient were tested: $\mathrm{k}=0.99$ (proposed model) and $\mathrm{k}=0.9999$ (standard model). The "true" coordinates were derived using Bernese software based on an 8-hour data set (Dach et.al., 2007). Figure 2 presents the results of 120 single epoch sessions processing the $31.2 \mathrm{~km}$ and $2.6 \mathrm{~km}$ baselines. The blue lines depict the linear residuals of the position obtained from single epoch processing, with respect to the "true" position from Bernese. The residuals were computed as: $V_{d}=\sqrt{\Delta X^{2}+\Delta Y^{2}+\Delta Z^{2}}$, where $\Delta \mathrm{X}, \Delta \mathrm{Y}$ and $\Delta \mathrm{Z}$ are components of the residuals with respect to the "true" position. The red lines depict the linear residuals of the a priori position, with respect to the "true" position. In most cases, a priori position was farther than $1 \mathrm{~m}$ from the 'true' position. The first column includes the results for $\mathrm{k}=0.99$. There were 87 correct (correct values of the ambiguities and linear residuals less than $10 \mathrm{~cm})$ among all 120 solutions $(72.5 \%)$ in the case of $31.2 \mathrm{~km}$ baseline. In the case of $2.6 \mathrm{~km}$ baseline there were 92 correct among all 120 solutions $(77 \%)$. The second column depicts the results for $\mathrm{k}=0.9999$. The results in this case are as follows: 38 correct among all 120 solutions (31.6\%) for $2.6 \mathrm{~km}$ baseline and $11 \mathrm{correct}$ among all 120 solutions $(9.2 \%)$ for $31.2 \mathrm{~km}$ baseline. The results show that the proposed in this contribution the assumption of $\mathrm{k}=0.99$ is much more advantageous than $\mathrm{k}=0.9999$ (which corresponds to the standard model with code observations). 

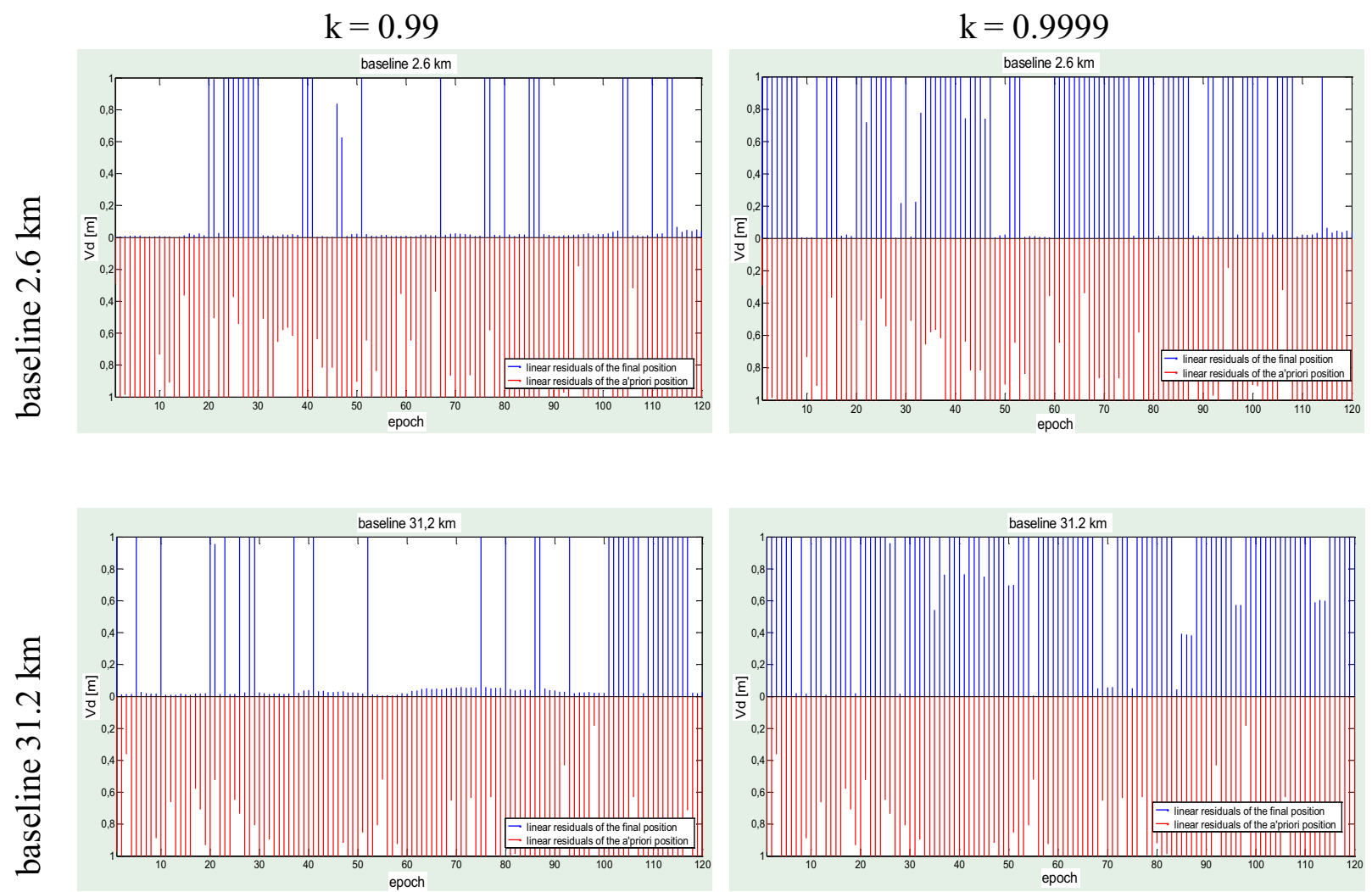

Fig. 2. The results of single-epoch processing of $31.2 \mathrm{~km}$ and $2.6 \mathrm{~km}$ baselines

\section{CONCLUSIONS}

The Lambda method of AR is based on the ambiguity VC matrix obtained from the float solution. OTF AR is performed on the basis of data from short sessions or even in extreme case from a single epoch. In such case, the VC matrix is not positive definite. In order to perform an integer decorrelation process, an estimator of the modified ambiguity variancecovariance matrix can be used. In the paper, the formula of such an estimator was derived. This estimator of the VC matrix was used in the cascade processing. The results of the tests show the usefulness of the proposed solutions. Nevertheless, more theoretical and experimental analyses are required in this subject. In the near future it is planned, among others, to develop an estimation method of the k coefficient.

\section{REFERENCES}

Bakula M. (2010) Network Code DGPS Positioning and Reliable Estimation of Position Accuracy, Survey Review, 42, 315, 82-91.

Cellmer S, Wielgosz P, , Rzepecka Z (2009) GNSS Carrier Phase Processing Using Modified Ambiguity Function Approach, Florence, Italy, May 27-30 2009, EUREF Publication, Mitteilungen des Bundesamtes für Kartographie und Geodäsie (in print).

Cellmer S, Wielgosz P , Rzepecka Z (2010) Modified ambiguity function approach for GPS carrier phase positioning, J Geod, Vol. 84, 264-275.

Cellmer S., (2011a): The real time precise positioning using MAFA method, The $8^{\text {th }}$ International Conference ENVIRONMENTAL ENGINEERING, selected papers, Vol. III, Vilnius, 1310-1314. 
Cellmer S. (2011b), Using the Integer Decorrelation Procedure to increase of the efficiency of the MAFA Method, Artificial Satellites, Vol. 46, No. 3, 103-110.

Cocard M and Geiger A (1992) Systematic Search for all Possible Widelanes. Proc. The Sixth International Geodetic Symposium on Satellite Positioning, Columbus Ohio March 1992, Vol. 17-20, 312-318.

Cocard M, Bourgon S Kamali O, Collins P (2008) A systematic investigation of optimal carrier-phase combinations for modernized triple-frequency GPS, J Geod, Vol. 82, 555-564.

Dach R, Hugentobler U, Fridez P, Meindl M ( 2007)BERNESE GPS Software Version 5.0., Astronomical Institute, University of Berne

Gui Q, Han S (2007) New algorithm of GPS rapid positioning based on double k-type ridge estimation. J Surv Eng 133(4):173-178

Han S and Rizos C (1996) Improving the computational efficiency of the ambiguity function algorithm. J Geod 1996, Vol. 70, No. 6, 330-341.

Henkel and Gunter (2007) Integrity Analysis of Cascaded Integer Resolution with Decorrelation Transformations. Proc. The 2007 National Technical Meeting of The Institute of Navigation January 22 - 24, 2007, 903-910

Hofmann-Wellenhof B, Lichtenegger H, Wasle E. (2008) GNSS-Global Navigation Satellite Systems - GPS, GLONASS, Galileo \& more, Springer-Verlag Wien

Joosten P (2001) The LAMBDA-Method: MatlabTM Implementation, Version 2.1 Mathematical Geodesy and Positioning, Civil Engineering and Geosciences, Delft University of Technology, The Netherlands,

Joosten P and Tiberius CCJM (2002) LAMBDA: FAQs. GPS Solutions, 6 (1-2), 109-114

Jung J and Enge P (2000) Optimization of Cascade Integer Resolution with Three Civil GPS Frequencies Proc. ION GPS'2000, Salt Lake City, September 2000

Kashani, I., Grejner-Brzezinska, D.A., and Wielgosz, P., (2005), Towards Instantaneous Network-Based RTK GPS Over 100 km Distance, Navigation, Vol. 52, No. 4, 239-245

Leick A (2004) GPS Satellite Surveying. $3^{\text {rd }}$ edition, John Wiley and Sons, Inc. 2004

Li B, Shen Y and Feng Y (2010) Fast GNSS ambiguity resolution as an ill-posed problem $J$ Geod, Vol. 84, No. 11, 683-698

Ou J, Wang Z (2004) An improved regularization method to resolve integer ambiguity in rapid positioning using single frequency GPS receivers. Chin Sci Bull 49(2):196-200

Shagimuratov, I.I., Baran, L.W., Wielgosz, P., and Yakimova, G.A., (2002), The structure of mid- and high-latitude ionosphere during September 1999 storm event obtained from GPS observations, Annales Geophysicae, Vol. 20, No 6, 665-671

Shen Y, Li B (2007) Regularized solution to fast GPS ambiguity resolution. J Surv Eng 133(4):168-172

Teunissen PJG (1993) Least squares estimation of the integer GPS ambiguities. Invited lecture, Section IV: theory and methodology. IAG General Meeting, Beijing

Teunissen P JG (1995) The least-squares ambiguity decorrelation adjustment: a method for fast GPS integer ambiguity estimation, $J$ Geod , 1995, Vol. 70, 65-82. 
Teunissen PJG, de Jonge PJ and Tiberius CCJM (1996) The volume of the GPS ambiguity search space and its relevance for integer ambiguity resolution, Proc. ION GPS '96 889898, Kansas City USA, 17-20 September 1996.

Teunissen PJG and Kleusberg A (1998) GPS for Geodesy, Springer - Verlag, Berlin Heidelberg New York, 1998

Teunissen PJG (1999) An optimality property of the integer least squares estimator, $J$ Geod 73:587-593

Urquhart L (2009) An Analysis of Multi-Frequency Carrier Phase Linear Combinations for GNSS, Senior technical report, Department of Geodesy and Geomatics Engineering Technical Report No. 263, University of New Brunswick, Fredericton, Canada, 71

Verhagen, S. (2005) "On the Reliability of Integer Ambiguity Resolution," Navigation, Vol. 52, No. 2, pp. 99-110.

Verhagen S. and Teunissen PJG (2006) New Global Navigation Satellite System Ambiguity Resolution Method Compared to Existing Approaches JOURNAL OF GUIDANCE, CONTROL, AND DYNAMICS Vol. 29, No. 4, July-August 2006 Delft University of Technology, 2629 HS Delft, The Netherlands

Wielgosz P (2011) Quality assessment of GPS rapid static positioning with weighted ionospheric parameters in generalized least squares, GPS Solutions Vol. 15, Issue 2, April 2011, 89-99

Xu P (2006) Voronoi Cells, Probabilistic Bounds, and Hypothesis Testing in Mixed Integer Linear Models, IEEE Transactions on Information Theory, 2006, Vol. 52, No. 7, 31223138 .

Received: 2012-03-21,

Reviewed: 2012-07-04,

Accepted: 2012-07-06. 